CDD: 120

\title{
LA PARADOJA DE FITCH Y LOS MUNDOS EPIS- TÉMICOS ABIERTOS
}

\author{
DIEGO TAJER \\ Universidad de Buenos Aires - CONICET \\ Argentina \\ diegotajer@gmail.com
}

Received: 22.08.2013; Revised: 8.04.2014; Accepted: 30.04.2014

\begin{abstract}
In this paper, I will provide a solution to Fitch's paradox which is based in the failure of epistemic closure. My approach will be semantical, following Beall (2009). Unlike his proposal, my system is logically classical, and relatively conservative with respect to statements about knowledge; all its deviations will be caused by the introduction of modal statements. In order to make this happen, I will introduce abnormal possible worlds (in this, I'm close to Beall), but also open worlds of two different kinds, which will explain the epistemic deviations of the agents.

Keywords: Fitch's paradox, Knowability, Open worlds, Anti-realism, Epistemic closure
\end{abstract}

Resumen: En este artículo, daré una solución a la paradoja de Fitch que se basa en el quiebre de la clausura epistémica. Mi enfoque será semántico, en la línea de Beall (2009). A diferencia de la propuesta de éste, mi sistema es lógicamente clásico, y relativamente conservador respecto a las afirmaciones sobre el conocimiento; todas sus desviaciones se basarán en la introducción de afirmaciones modales. Para dar lugar a ello, introduciré mundos posibles anormales (en esto, me acerco a Beall), pero además, mundos abiertos de dos tipos distintos que funcionarán como explicación de las desviaciones epistémicas de los agentes.

Palabras-claves: Paradoja de Fitch, Cognoscibilidad, Mundos abiertos, Antirealis-mo, Clausura epistémica

Manuscrito - Rev. Int. Fil., Campinas, v. 37, n. 1, p. 85-116, jan.-jun. 2014. 


\section{El acusado y la estrategia de la defensa}

La paradoja de Fitch puede resumirse así: dados algunos supuestos bastante inocentes de lógica modal y epistémica (por ejemplo, Necesitación modal, Factividad y Distribución de K sobre la conjunción ${ }^{1}$ ), podemos deducir que si toda verdad es cognoscible, entonces toda verdad es conocida. La estructura tradicional de la argumentación es esta ${ }^{2}$. Supongamos que vale el principio de cognoscibilidad $(\vdash A \rightarrow \diamond K A)$, y que A es una verdad no conocida:

1. A $\wedge \neg K$ A [Supuesto]

2. $\mathrm{K}(\mathrm{A} \wedge \neg K \mathrm{~A})[$ Supuesto]

3. KA [2, Distribución de K sobre conjunción]

4. $\mathrm{K} \neg K \mathrm{~A}[2$, Distribución de $\mathrm{K}$ sobre conjunción]

5. $\neg \mathrm{KA}[3$, Factividad de K]

6. $\perp[3,5, \neg$-Elim $]$

7. $\neg \mathrm{K}(\mathrm{A} \wedge \neg K \mathrm{~A})[2-6, \neg$-intro]

8. $\square \neg \mathrm{K}(\mathrm{A} \wedge \neg K \mathrm{~A})[7$, Necesitación de $\square]$

9. $\neg \diamond \mathrm{K}(\mathrm{A} \wedge \neg K \mathrm{~A})[8$, Interdefinibilidad modal $]$

10. $\diamond \mathrm{K}(\mathrm{A} \wedge \neg K \mathrm{~A})$ [1, Principio de cognoscibilidad]

11. $\perp[9,10, \neg$-Elim $]$

12. $\neg(A \wedge \neg K A)[1-11, \neg$-Intro]

13. $A$ [Supuesto]

\footnotetext{
${ }^{1}$ La paradoja puede surgir de menos elementos que estos. Parte del debate en el que no entraré es qué principios son necesarios para derivarla.

${ }^{2}$ Usaré los símbolos K y $\square$ como operadores y no como predicados. Esta elección está relacionada con el enfoque semántico que adoptaré luego.
} 
14. $\neg K A$ [Supuesto]

15. $A \wedge \neg K A[13,14, \wedge-$ Intro $]$

16. $\perp[12,15, \neg$-Elim $]$

17. $\neg \neg K A[12-16, \neg-$ Intro $]$

18. $K A[17$, Doble Negación]

19. $A \rightarrow K A[13-18, \rightarrow$-Intro]

El debate alrededor de la paradoja es fecundo pero no necesariamente encaminado a la solución de ella. Algunos (por ejemplo, Jenkins 2009) sostienen que la paradoja es una reducción al absurdo del principio de cognoscibilidad, y con él, del anti-realismo. Es decir, sólo quedarían afectados quienes sostienen que toda verdad es cognoscible, pero la paradoja no ofrece ningún tipo de problema (más allá de la posible sorpresa) a quienes sostienen que hay verdades incognoscibles, y por ende no es necesario "solucionarla". De todas maneras, es generalmente admitido que la paradoja es bastante inocente para los anti-realistas más comunes, que suelen rechazar la lógica clásica. Si uno utiliza una lógica intuicionista (como defiende Dummett (2009)), no puede llegar al resultado de que toda verdad cognoscible es conocida o en otras palabras, que para toda oración A, se da que $A \rightarrow K A$. Sólo puedo llegar a un resultado mucho más débil, esto es, que $A \rightarrow \neg \neg K A$ (algo así como "si A es verdadera, es absurdo que sea absurdo conocer A"), para todas las oraciones A, lo cual no parece demasiado polémico si uno admite que A es cognoscible. De este modo, no está claro que los anti-realistas tradicionales puedan verse realmente afectados por la paradoja ${ }^{3}$.

\footnotetext{
${ }^{3}$ El punto es obviamente discutible. Percival (1990) sostiene que la paradoja de Fitch es también problemática para los intuicionistas porque, incluso si no usáramos doble negación, llegaríamos a la discutible conclusión de que $\neg K A \rightarrow \neg A$.
}

Manuscrito - Rev. Int. Fil., Campinas, v. 37, n. 1, p. 85-116, jan.-jun. 2014. 
Sin embargo, otros anti-realistas se ven afectados de manera más clara por la paradoja. El defensor contemporáneo más arduo del antirealismo, Neil Tennant (1997), considera que uno puede sostener el principio de cognoscibilidad en el marco de la lógica clásica. Esta posición es calificada como "Optimismo Godeliano" o anti-realismo suave, y se basa en la aceptación de la lógica clásica pero el rechazo, ya sea por motivos dummettianos (como la necesidad de manifestación del significado) o metafísicos, de la idea de una verdad trascendente al reconocimiento. Tanto Tennant como Edgington (1982), por ejemplo, enfocaron sus soluciones a este tipo de anti-realismo, y no al típicamente dummettiano (es decir, intuicionista). La intención de este artículo será entonces defender una versión del anti-realismo suave (de ahora en más, anti-realismo a secas) que sea filosóficamente coherente.

Determinado el "acusado" de la paradoja (el anti-realismo suave), queda ahora por determinar la estrategia que tomaré para solucionarla (que he llamado "la estrategia de la defensa"). Las salidas tradicionales a la paradoja de Fitch suelen ir por dos carrilles: por un lado, se pueden proponer restricciones para el principio de cognoscibilidad. La propuesta más importante en esta familia es la de Tennant (1997). $\mathrm{Su}$ idea es sostener un anti-realismo relativamente restringido, donde todas las verdades sean cognoscibles salvo algunas que por principio escapan a esta condición (por ejemplo, $A \wedge \neg K A$ ). Williamson (2009) criticó fuertemente esta salida, en particular por proponer una excepción ad hoc a un principio fundamental para el anti-realismo como la cognoscibilidad. Según este autor, Tennant no puede explicar satisfactoriamente, en un marco filosóficamente anti-realista, por qué algunas oraciones sí pueden ser verdaderas sin ser cognoscibles.

En paralelo a las estrategias de restricción, existe otro camino para evitar la paradoja: debilitar el resto de los principios que permiten la argumentación. Si bien no está claro, como antes señalé, qué principios son estrictamente necesarios para su obtención, sí sabemos de 
muchos que son suficientes (por ejemplo, Necesitación, Distributividad y Factividad). Este segundo tipo de estrategias busca proponer y justificar debilitamientos para estos principios, y diseñar nuevos sistemas de lógica epistémica modal que bloqueen la inferencia de Fitch.

En este artículo tomaré una estrategia del segundo tipo. Por las razones aducidas por Williamson (2009), me resulta inadecuado defender el anti-realismo restringiendo la cognoscibilidad. Propondré una solución basada en el debilitamiento de otros principios: en particular, el principio de Necesitación modal. Según mi postura (que considero compatible con el anti-realismo en general), hay dos tipos de mentes, unas empíricamente posibles y otras metafísicamente posibles (obviamente el primer conjunto está incluido en el segundo). Si bien la distributividad del conocimiento no puede fallar para mentes empíricamente posibles, esta ley no vale en su generalidad; es decir, ciertas mentes metafísicamente posibles pueden conocer una conjunción sin conocer los conyuntos. Son justamente esas mentes las que pueden conocer "A y A no es conocida" sin entrar en contradicción.

Mi planteo está inspirado en el de Beall (2009), cuyo enfoque expondré y criticaré en la sección 2 . En la sección 3, expondré mi propuesta en sus detalles filosóficos y formales. En la sección 4 analizaré los principales resultados del sistema formal propuesto, y justificaré cada uno de ellos.

\section{La propuesta paracompleta de Beall}

En esta sección expondré la propuesta paracompleta de Beall (2009), que en mi opinión es la manera más interesante de bloquear la paradoja de Fitch, y plantearé las objeciones que me llevarán a mi propia propuesta (desarrollada en la próxima sección).

Ante todo, algunas aclaraciones terminológicas. Llamamos paracompleto a un sistema que no tiene a $A \vee \neg A$ como teorema. Llama- 
mos paraconsistente a un sistema donde no vale la regla Ex falso. En su artículo, Beall desarrolla tres teorías distintas para responder a la paradoja de Fitch. Aquí tomaré en cuenta particularmente la segunda de ellas. Brevemente explicaré por qué elijo no desarrollar las otras propuestas. Tanto en la primera como en la tercera, que son en algún sentido paraconsistentes, la estrategia de Beall es similar a la desarrollada por Priest (2009): postular, para cada verdad A, un mundo modalmente accesible donde la conjunción $A \wedge \neg A$ sea verdadera. En la primera propuesta $^{4}$, este mundo es trivial, por lo cual toda afirmación es verdadera en él (incluyendo, obviamente, $K A$ para todas las verdades A). En la tercera propuesta, este mundo es solamente inconsistente, y tiene (por estipulación) la característica de acceder epistémicamente sólo a sí mismo, por lo cual (siguiendo las cláusulas tradicionales de la lógica epistémica) la conjunción $K(A \wedge \neg A)$ también será verdadera en él. Así, en ambas propuestas, siguiendo las cláusulas tradicionales de la lógica modal, se logra fácilmente obtener el Principio de Cognoscibilidad: para cada verdad A en w, hay un mundo modalmente accesible donde $K A$ (y, aunque no sea relevante, también $K \neg A$ ) es verdadero, y por ende $\diamond K A$ es verdadero en $\mathrm{w}$.

Lo bueno de estas propuestas es que preservan el Principio de Cognoscibilidad, junto con otros principios importantes como la Factividad de K y su Distributividad bajo conjunción. Sin embargo, el principal problema de estas propuestas es manifiesto: para cualquier verdad A en un mundo, la afirmación $\diamond(A \wedge \neg A)$ es verdadera en él. Esto es, se debe postular que, para cualquier verdad A, es posible la contradicción $A \wedge \neg A$. Eso nos lleva muy lejos del anti-realismo, que si bien sostiene cierta tensión con principios clásicos como el Tercero Excluído, manti-

\footnotetext{
${ }^{4}$ Esta primera propuesta es prácticamente la misma que la de Priest (2009), y si bien no tiene aspectos estrictamente paraconsistentes (pues el único mundo inconsistente es de hecho trivial), sí tiene una concepción totalmente heterodoxa de la posibilidad (según la cual un mundo trivial es posible).
} 
ene a rajatabla la ley de no contradicción tanto en su versión actualista como modal (es decir, que es imposible que una contradicción tenga lugar). Para sostener la posibilidad de (prácticamente) toda contradicción, hace falta dar razones independientes, que parecen estar en contraposición con los principios anti-realistas tradicionales.

Otros problemas pertenecen a cada propuesta en particular. En la primera, la presencia de un mundo trivial es completamente misteriosa: es difícil dar una explicación filosóficamente satisfactoria de la posibilidad de un mundo como ese (de hecho, Beall ni intenta hacerlo), y mucho menos contar una historia en la cual la posible trivialidad salve a un planteo anti-realista tradicional. En la tercera propuesta queda sin explicar por qué los mundos inconsistentes sólo pueden acceder epistémicamente a sí mismos. Este aspecto de la semántica resulta completamente ad hoc, y parece inventado sólo para salvar el Principio de Cognoscibilidad. Además, no queda claro qué estatus metafísico tienen los mundos inconsistentes y por qué supuestamente el anti-realista apela a ellos cuando dice que toda verdad es cognoscible. Para el grueso de la tradición metafísica, es muy cuestionable un concepto de posibilidad que permita contradicciones; se supone que la ley de no-contradicción es uno de los principios paradigmáticamente necesarios.

La segunda propuesta de Beall es la que tomaré en cuenta en este artículo. El autor la caracteriza como meramente paracompleta, pues en ella no vale el Tercero Excluido. La razón teórica de la falla de esta verdad clásica es simplemente que el sistema está planteado en $K_{3}$, la lógica de Lukasiewicz, donde no sólo es inválido el Tercero Excluido, sino también toda verdad lógica ${ }^{5}$.

La elección de $K_{3}$ como lógica de base es discutible. Beall apela a

\footnotetext{
${ }^{5} K_{3}$ es una lógica trivalente normal (es decir, a inputs clásicos da outputs clásicos), donde la conjunción entre .5 y 1 da 0.5 y entre .5 y 0 da 0 ; la disyunción entre .5 y 0 da .5 y entre .5 y 1 da 1 ; el condicional con antecedente 0 o consecuente 1 vale $1, .5 \rightarrow 0$ vale .5, y $1 \rightarrow .5$ vale .5
} 
ella simplemente porque cree que una verdadera solución a la paradoja de Fitch debe responder también a la Paradoja del Conocedor, y que las opciones paracompletas son las únicas no paraconsistentes que pueden hacerlo. La Paradoja del Conocedor consiste en una oración obtenida por Diagonalización que dice de sí misma "yo no soy conocida". Si K es factivo y vale necesitación, podemos llegar a una contradicción:

1. $A \leftrightarrow \neg K(\langle A\rangle)$ [Lema de diagonalización]

2. $K(\langle A\rangle)[$ Supuesto]

3. A $[2$, Factividad $]$

4. $\neg K(\langle A\rangle)[1,3$, Modus Ponens $]$

5. $\perp[3,4, \neg$-Elim $]$

6. $\neg K(\langle A\rangle)[2-5, \neg-$ Intro $]$

7. $A[1,6$, Modus Ponens $]$

8. $K(\langle A\rangle)[7$, Necesitación epistémica]

9. $\perp[2,6, \neg$-Elim $]$

Hay un aspecto muy llamativo de la apelación de Beall a $K_{3}$. El autor parece decir que la suposición de fondo para obtener la paradoja es que la oración del conocedor es conocida o no (2009, p. 108). Otra posibilidad es que la oración misma no sea verdadera ni falsa. Sin embargo, de ninguna de las dos maneras se obtendría $A \leftrightarrow \neg K(\langle A\rangle)$ como verdad en $K_{3}$. Si el valor de A (o de $K(\langle A\rangle)$ ) es intermedio, el valor de $\neg A$ (o de $\neg K(\langle A\rangle))$ también lo es. De este modo, sea cual sea el valor de $K(\langle A\rangle)$ (o de $\mathrm{A}$ ), el bicondicional resultará ni verdadero ni falso ${ }^{6}$. No está para nada claro, entonces, que asumir que un sujeto ni conoce ni desconoce una proposición nos salve de la paradoja.

\footnotetext{
${ }^{6}$ Por simetría, probaré el resultado para A. Se aplica exactamente igual para $\neg K(\langle A\rangle)$. Supongamos que $v(A)=.5$. Entonces por las cláusulas de $K_{3}$, hay tres opciones. La primera es que $v \neg K(\langle A\rangle)=1$. En ese caso,
} 
Por lo tanto, Beall quizás está pensando en una versión de $K_{3}$ donde no vale Necesitación o Factividad. Pero en ese caso, no hace falta irse tan lejos: es posible atacar la Necesitación sin adoptar sistemas paracompletos. De hecho, eso es lo que haré en lo siguiente.

Para hacer más fácil la comparación, ya que he objetado la paracompletitud de la propuesta de Beall, la expondré en lógica clásica y no en $K_{3}$. En el planteo de Beall, hay dos tipos de mundos: Normales y Anormales. En todos los mundos, los conectivos y los operadores modales se comportan tradicionalmente. Sin embargo, el operador epistémico K se comporta como es tradicional en los mundos normales, y arbitrariamente en los mundos anormales. Esto es, los mundos anormales justamente varían en su comportamiento de $\mathrm{K}$.

Las relaciones de accesibilidad metafísica (R) y epistémica (E) son reflexivas. Asimismo, los modelos incluyen mundos "narcisistas", esto es, mundos que sólo acceden epistémica y metafísicamente a sí mismos. Para obtener Cognoscibilidad, se postula que toda verdad en un mundo normal es conocida en un mundo anormal narcisista metafísicamente accesible desde éste. Para evitar inconsistencias, también se postula que el operador $\mathrm{K}$ en mundos anormales es factivo.

Como es costumbre en las propuestas no-normales, la validez es definida como preservación de verdad en mundos normales. El sistema permite un contraejemplo para la paradoja de Fitch.

(En este artículo, daré modelos de estructuras modales con gráficos.

$\overline{v(\neg K(\langle A\rangle) \rightarrow A)=.5 \text { y } v(A} \rightarrow \neg K(\langle A\rangle))=1$, por lo cual $v(\neg K(\langle A\rangle) \leftrightarrow$ $A)=.5$ dado que $v(\neg K(\langle A\rangle) \leftrightarrow A)=v((\neg K(\langle A\rangle) \rightarrow A) \wedge(A \rightarrow \neg K(\langle A\rangle)))$, y que el valor de una conjunción con verdadero e indeterminado es indeterminado. La segunda opción es que $v(\neg K(\langle A\rangle))=0$. En ese caso, $v(\neg K(\langle A\rangle) \rightarrow A)=.5$ y $v(A \rightarrow \neg K(\langle A\rangle))=.5$, por lo cual $v(\neg K(\langle A\rangle) \leftrightarrow$ $A)=.5$ dado que el valor de una conjunción con verdadero e indeterminado es indeterminado. La tercera posibilidad es que $v(\neg K(\langle A\rangle))=.5$. En ese caso, $v(\neg K(\langle A\rangle) \rightarrow A)=.5$ y $v(A \rightarrow \neg K(\langle A\rangle))=.5$, por lo cual $v(\neg K(\langle A\rangle) \leftrightarrow A)=.5$ dado que el valor de una conjunción con dos interminados es indeterminado.

Manuscrito - Rev. Int. Fil., Campinas, v. 37, n. 1, p. 85-116, jan.-jun. 2014. 
La flecha $R$ indica accesibilidad metafísica, la flecha $\mathrm{E}$ indica accesibilidad epistémica, y las oraciones entre paréntesis son algunas oraciones relevantes que el mundo en cuestión hace verdaderas. Dado que las relaciones de accesibilidad son reflexivas, omito las flechas de cada mundo hacia sí mismo, que se suponen presentes en todos los casos. En algunos casos, usaré la terminología $A$, para indicar que la fórmula $A$ no es verdadera en un determinado mundo (lo que no implica, en determinados contextos, que sea falsa en ese mundo). Los mundos tendrán subíndices $N$ o $A$, que indican si se trata de un mundo normal o anormal.)

En el siguiente modelo, en el mundo $w_{N}$, p es verdadera pero desconocida.

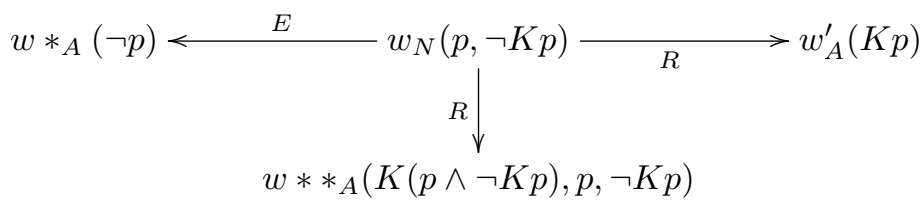

Como puede verse, el núcleo del contramodelo es que existe un mundo $\left(\mathrm{w}^{* *}\right)$ donde el operador epistémico no se distribuye bajo conjunción. La idea de estos mundos anormales, entonces, es que en ellos el operador $K$ se comporta de manera desviada.

La propuesta de Beall es interesante y, hasta cierto punto, efectiva. Sin embargo, creo que no ayuda demasiado para responder de manera satisfactoria al planteo de la paradoja.

En primer lugar, por tener a $K_{3}$ como lógica subyacente (aunque la versión que expuse, repito, es bivalente). Esto hace que su conexión con el anti-realismo sea discutible: es cierto que un anti-realista rechaza el Tercero Excluido, pero es muy raro que rechace la Ley de No Contradicción o la Ley de Identidad (que fallan, como toda otra verdad lógica, en $K_{3}$ ).

En segundo lugar, porque el comportamiento desviado de $\mathrm{K}$ tiene que responder a alguna razón filosófica de peso. Esto es, no basta con 
una asignación arbitraria de valuaciones a las afirmaciones $\mathrm{K}$ en mundos anormales "narcisistas". El narcisismo de esos mundos, así como su anormalidad, requiere alguna explicación, para no resultar ad hoc. Y creo que ella se puede dar en el plano semántico.

Por otro lado, no está claro en qué sentido lo obtenido beneficia al anti-realismo. Pues la propuesta reduce el principio de cognoscibilidad a la mera idea de que toda verdad es cognoscible en algún mundo donde el conocimiento, más allá de la factividad, no tiene ningún rasgo de los que solemos atribuirle. Como el mismo Beall admite, si tenemos dos tipos de "conocimiento" distintos, el verificacionista sigue con el problema de antes si postula que todo lo verdadero es cognoscible en el sentido tradicional del concepto 'conocer'.

En el próximo apartado expondré mi propuesta, que intenta solucionar los problemas de la de Beall, y en particular, tener un planteo filosóficamente más sólido que pueda conectarlo con la tradición antirealista y decirnos algo sobre el sentido de la paradoja de Fitch.

\section{Mi propuesta: mundos raros y abiertos}

Cuando los anti-realistas sostienen la cognoscibilidad, en general admiten que no están refiriéndose a un tipo estrictamente humano de cognoscibilidad, sino a alguna clase de extensión finita de los poderes humanos (Wright 1982). Esto se debe a que si lo humano es la medida, la posición es difícil de defender: hay numerosas verdades que ningún humano puede conocer debido a los límites de sus capacidades naturales, y es demasiado radical afirmar que ellas no son verdades realmente (es la posición que tienen los llamados 'finitistas estrictos'). En general los anti-realistas apelan a algún tipo de idealización de las capacidades humanas.

La lógica epistémica tradicional también hace uso de idealizaciones. La más llamativa de ellas es la clausura epistémica. Dado el 
marco kripkeano de la semántica de la lógica epistémica, tenemos que postular que cuando alguien conoce una oración, conoce también todas sus consecuencias lógicas; como corolario, todos conocemos todas las verdades lógicas (omnisciencia lógica). No hay demasiadas dudas sobre que esto es empíricamente falso; por ejemplo, muchos sabemos los cinco axiomas de la aritmética de Peano sin por eso conocer todas sus consecuencias lógicas.

En mi propuesta, a diferencia de la de Beall, esta idealización no tiene lugar. Esto no es una diferencia menor. Pues creo que para defender una lógica epistémica determinada, hace falta tener bien claro que ella intenta modelar el conocimiento tal como realmente es, y no un concepto ideal que existe en el cielo platónico. En ese sentido, el conocimiento será "desviado" no sólo en mundos anormales, sino también en los mundos normales, como creo que es nuestro mundo.

Mi propuesta está basada en los siguientes desiderata:

1. Que la lógica sea bivalente. Para que el sistema pueda constituir una verdadera defensa del anti-realismo suave y no un ejemplo de juguete.

2. Que los teoremas respecto al conocimiento reflejen en la mejor medida posible lo que sucede realmente con el conocimiento en agentes reales. Por eso, fallará la clausura epistémica.

3. Que sean válidas las leyes lógicas y algunas generalizaciones sobre el comportamiento del operador K en mundos normales (a las que llamaré leyes epistémicas').

4. Que el conocimiento sea definido del mismo modo en todo mundo posible, para evitar arbitrariedades.

5. Que se admita la existencia de sujetos metafísicamente posibles, con capacidades lógicas muy inferiores a las de un sujeto normal o empírico. La inspiración para esto es (Beall 2009), que sin embargo no cumple con los anteriores cuatro desiderata. 
Para dar lugar a la falla de la clausura epistémica, siguiendo a Priest $(2006)^{7}$, haré uso de "mundos" abiertos. Un mundo abierto es un "mundo" donde algunas leyes lógicas pueden fallar. Por eso, muchos muchos mundos abiertos son metafísicamente imposibles. La idea es la siguiente: dado que es posible saber los axiomas de Peano y no una verdad matemática derivada de ellos, habrá un mundo, no posible, sino abierto o meramente representacional, donde los axiomas de Peano sean verdaderos pero esa verdad matemática no lo sea.

Siguiendo el planteo anti-realista, habrá dos tipos de mundos posibles. Los mundos posibles normales son empíricamente posibles, y en ellos habrá cierto nivel de clausura epistémica ${ }^{8}$; pues no considero que sea empíricamente posible, por ejemplo, conocer una conjunción pero no conocer los conyuntos. Los mundos posibles anormales, por otro lado, pueden incluir a sujetos o mentes que son meramente posibles en un sentido metafísico, no empírico. En los mundos posibles anormales, la clausura epistémica puede fallar de manera absoluta, porque las mentes metafísicamente posibles pueden tener una capacidad lógica muy débil.

Correspondiendo con esta distinción, haré uso de dos tipos de mundos abiertos para dar a entender dos niveles, uno empírico y otro meramente posible, de tener fallas epistémicas. Los mundos abiertos normales son las representaciones que los sujetos empíricos pueden hacerse, y por ello tienen cierta estructura lógica. En cambio, los mundos abiertos anormales son las representaciones que sujetos meramente posibles (en sentido metafísico) pueden tener; por esa razón, en estos "mundos" las leyes lógicas pueden fallar de manera arbitraria.

Por ende, habrá cuatro tipos de mundos:

\footnotetext{
${ }^{7}$ Vale aclarar que la utilización de mundos abiertos o imposibles para anular la clausura, si bien no es popular, es de larga data.

${ }^{8}$ Llamo clausura epistémica a la propiedad de que si un sujeto conoce un conjunto de enunciados, también conoce las consecuencias lógicas de esos enunciados.
} 
1. Mundos posibles. En ellos, las tautologías de la lógica clásica y la lógica modal son verdaderas siempre.

- Normales: Las leyes epistémicas (tautologías que incluyen al operador K) son verdaderas en ellos.

- Anormales: Las leyes epistémicas pueden fallar en ellos.

2. Mundos abiertos. Representan las alternativas epistémicas de los agentes. Dado que los agentes no son perfectamente racionales, algunas tautologías de la lógica clásica o modal pueden fallar aquí.

- Normales: Representan las alternativas epistémicas de agentes normales. Están lógicamente estructurados hasta cierto punto.

- Anormales: Representan las alternativas epistémicas de agentes anormales (i.e. meramente posibles). Por ello, pueden carecer de estructura lógica.

Ahora los detalles formales. Llamaré $P_{N}$ y $P_{A}$ al conjunto de mundos posibles normales y anormales respectivamente; y llamaré $O_{N}$ y $O_{A}$ al conjunto de mundos abiertos normales y anormales respectivamente.

Sea $\mathrm{V}$ el conjunto de valores de verdad $\{0,1\}$. Una interpretación es una estructura $<v, W, P, P_{N}, P_{A}, O, O_{N}, O_{A}, R, E>$ para un lenguaje $\mathrm{L}$, siendo $\mathrm{R}$ y E relaciones reflexivas en $\mathrm{W}, R \cap(P \times O)=\emptyset$ (es decir, desde ningún mundo posible puede acceder metafísicamente a un mundo abierto), $E \cap\left(P_{N} \times O_{A}\right)=\emptyset$ (i.e. ningún mundo posible normal puede acceder epistémicamente a mundos abiertos anormales) donde $P_{N} \cup P_{D}=P, O_{N} \cup O_{A}=O, P \cup O=W$, y $v$ es una función $L \times W \longrightarrow V$ tal que:

- Para todo $w \in P$

$$
-v_{w}(A \wedge B)=1 \text { sii } v_{w}(A)=1 \text { y } v_{w}(B)=1
$$




$$
\begin{aligned}
& -v_{w}(A \vee B)=1 \text { sii } v_{w}(A)=1 \text { o } v_{w}(B)=1 \\
& -v_{w}(\neg A)=1 \text { sii } v(A)=0 \\
& -v_{w}(K A)=1 \text { sii para todo }{ }^{*} \text { tal que } \mathrm{wEw}^{*}, v_{w *}(A)=1 \\
& -v_{w}(\square A)=1 \text { sii para todo } \mathrm{w}^{*} \text { tal que } \mathrm{wRw}^{*}, v_{w *}(A)=1
\end{aligned}
$$

- Para todo $w \in O_{N}$,

$$
\begin{aligned}
& -v_{w}(A \wedge B)=1 \text { sii } v_{w}(A)=1 \text { y } v_{w}(B)=1 \\
& -v_{w}(A \vee B)=1 \text { sii } v_{w}(A)=1 \text { o } v_{w}(B)=1 \\
& -v_{w}(K A)=1 \text { sii para todo } \mathrm{w}^{*} \text { tal que } \mathrm{wEw}^{*}, v_{w *}(A)=1 \\
& -v_{w}(\square A)=1 \text { sii para todo } \mathrm{w}^{*} \text { tal que } \mathrm{wRw}^{*}, v_{w *}(A)=1 \\
& -v_{w}(\neg A)=1 \text { sólo si } v(A)=0^{9}
\end{aligned}
$$

- Para todo $w \in O_{A}$, la asignación de valores de verdad a las oraciones es arbitraria.

Por último, para salvar el Principio de Cognoscibilidad:

Cognoscibilidad Para todo $w \in P_{N}$, si $v_{w}(A)=1$, entonces existe un mundo $w * \in P$ tal que $\mathrm{wRw}^{*} \mathrm{y} v_{w *}(K A)=1$

Validez $\Delta \vDash \mathrm{B}$ sii en todo $w \in P_{N}$ tal que para todo $\delta \in \Delta$ tenemos que $v_{w}(\delta)=1$, se da que $v_{w}(B)=1$ (i.e. validez es entendida como preservación de verdad en mundos posibles normales)

Llamaré leyes lógicas a aquellos teoremas o inferencias válidas en esta lógica que no incluyen al operador K. Llamaré leyes epistémicas a aquellos teoremas o inferencias válidas en esta lógica que sí incluyen a este operador.

\footnotetext{
${ }^{9}$ Esto permite que en los mundos abiertos normales sean posibles los vacíos de verdad. La elección de cláusulas semánticas para los mundos abiertos normales tiene cierto nivel de arbitrariedad. Lo que aquí puedo reflejar es que, como muchos casos históricos indican (Brouwer y Lukasiewicz, por ejemplo), algunos sujetos empíricamente posibles pueden ignorar $A \vee \neg A$.
} 


\section{Algunos resultados y sus justificaciones filosóficas}

En este apartado desarrollaré algunos de los resultados interesantes del sistema propuesto.

1. Aquí fallará la omnisciencia lógica, y con ella la clausura epistémica. Sin embargo, algunas leyes epistémicas tradicionales resultan válidas.

Clausura bajo introducción de disyunción. Sea w un mundo normal donde KA es verdadero. Supongamos que allí $K A \rightarrow K(A \vee B)$ es falso (definimos el condicional a la manera clásica en mundos normales). Entonces allí es verdadero el antecedente y falso el consecuente. Es decir, hay un mundo abierto normal (o posible) epistémicamente accesible $\mathrm{w}^{*}$ donde $A \vee B$ es falso. Allí, A es falso y B también. Pero dado que es accesible desde $\mathrm{w}$, por las cláusulas de $\mathrm{K}$, en $\mathrm{w}^{*}$ debe ser verdadero que A. Absurdo.

Contramodelo para Conocimiento de Tercero Excluído. (los gráficos deben leerse como antes indiqué; aquí agrego a cada mundo un subíndice $O_{N}, O_{A}, P_{N}$ о $P_{A}$ para indicar de qué clase de mundo se trata). Véase el mundo $w$ en este modelo.

$$
w_{P_{N}}(\neg K(p \vee \neg p)) \underset{E}{\longrightarrow} w *_{O_{N}}(\not p, \neg p)
$$

Justificación: Lo que se logra, así, es pasar de un modelo completamente idealizado del conocimiento a un modelo mucho más realista, donde no todas las verdades lógicas son conocidas por todo sujeto empírico. 
Asimismo, esto ayuda a hacer un sistema más coherente. Ya no hay mundos ideales (los mundos "normales" de Beall) donde el conocimiento está cerrado bajo deducción, y mundos "anormales" donde el conocimiento es arbitrario. Por el contrario, en los mundos posibles normales el conocimiento funciona de manera no ideal. Y en los mundos posibles anormales la diferencia ya no es cualitativa sino de grado: la imperfección de los sujetos que conocen se vuelve mucho más notoria en ellos.

2. Vale necesitación modal para oraciones sin K. En cambio, falla para las oraciones con K.

Necesitación para oraciones sin K. Sea w un mundo normal. Sea A una oración válida en el sistema que no contiene apariciones de K. Para todo $w \in P_{N}$, $v_{w}(A)=1$. Supongamos que existe un $\mathrm{w}^{*}$ posible normal tal que $v_{w *}(\square A)=0$. Entonces hay un $\mathrm{w}+$ tal que $\mathrm{w}^{*} \mathrm{Rw}+\mathrm{y} v_{w+}(A)=0$. Pero esto no puede ser, ya que por definición, los mundos posibles sólo acceden metafísicamente a otros mundos posibles, y en todos ellos los conectivos tienen el mismo funcionamiento. Absurdo.

Falla de Necesitación para oraciones con $K$. Antes hemos visto que $K A \rightarrow K(A \vee B)$ es un teorema en el sistema. En el siguiente modelo, la oración 
$\square(K p \rightarrow K(p \vee q))$ falla en $w$.

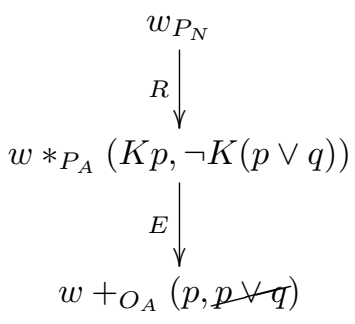

Justificación: este resultado puede ser polémico, ya que suele sostenerse que "los teoremas son necesarios" y que por ende Necesitación debe valer (así lo presenta Salerno 2009). Sin embargo, creo que esta forma de leer Necesitación es un engaño que provoca la misma idea de "lógica epistémica". De ninguna manera las verdades sobre el conocimiento, incluso en lógica epistémica, son verdades lógicas. Para eso necesitarían una neutralidad al tópico de la que carecen completamente. Estas verdades son como las de cualquier otra teoría, sólo que hemos elegido, por facilidad, expresarlas u obtenerlas en un sistema formal.

La Necesitación generalizada dice, en sentido estricto, que todas las leyes del conocimiento son necesarias. La mejor manera de defender este punto es arguir que que son verdades conceptuales, por lo cual la necesariedad va de suyo. Hay un sentido en que ciertamente es imposible que alguien sepa A y B pero no sepa A o no sepa B. El problema es cuál es el ese sentido. En mi propuesta, las leyes del conocimiento son como las leyes empíricas. Esto es, si bien es difícil concebir o imaginar que sean distintas (así como es difícil imaginar un mundo donde el agua sea veneno), debe admitirse por razones teóricas que ello es, sin embargo, completamente posible en un sentido metafísico. Entonces, si nos parece imposible que en un caso el conocimiento no se distribuya 
bajo conjunción, esto es porque nunca hemos visto ni podemos imaginarnos cómo sería eso. Pero lo mismo sucede con el quiebre de otras leyes empíricas, que sin embargo consideramos posible. Con el próximo resultado esto será profundizado.

3. Vale la distribución de $\mathrm{K}$ bajo conjunción, aunque no es necesariamente verdadera ${ }^{10}$.

Distribución de $K$ bajo conjunción. Sea w un mundo posible normal tal que $v_{w}(K(A \wedge B))=1$ pero $v_{w}(K A \wedge$ $K B)=0$. Entonces $v_{w}(K A)=0$ o $v_{w}(K B)=0$. Por simetría, suponemos que $v_{w}(K A)=0$. Si esto sucede, entonces hay un mundo abierto normal (o posible) $\mathrm{w}^{*}$ tal que $\mathrm{wEw}^{*}$ y $v_{w *}(A)=0$. Sin embargo, dado que $\mathrm{wEw}^{*}, v_{w *}(A \wedge B)=1$. Por ende, $v_{w *}(A)=1$. Absurdo.

No necesariedad de la distribución de $K$ bajo conjunción. En el siguiente modelo, $\square(K(p \wedge q) \rightarrow(K p \wedge$

\footnotetext{
${ }^{10}$ Algo similar, aunque sin pensar en términos de "fallas posibles", planteaba Church en su referato a Fitch (el texto aparece en Salerno 2009), para quien la paradoja de Fitch necesitaba clausura epistémica, y ésta solía fallar dado que "hay gente tonta". El hecho de que lo único necesario finalmente fuera la inocente distributividad de $\mathrm{K}$ bajo conjunción hace dudar de que la cierta existencia de gente tonta pueda tener algún efecto sobre la paradoja. En mi propuesta no hay gente tan "tonta" en el mundo actual, pero sí en otros mundos posibles.
} 
$K q)$ ) falla en $w$.

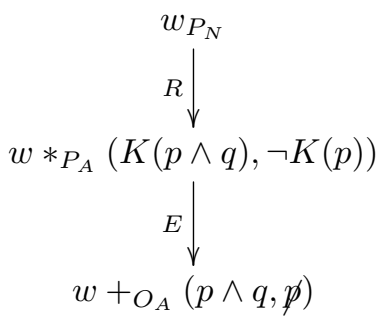

Justificación: este resultado va de la mano con el anterior. La idea de mi propuesta no es anular todas las leyes epistémicas, sino pensar la lógica epistémica como una teoría empírica sobre el conocimiento. Algunas leyes serán válidas por no fallar jamás en este mundo, como la distributividad de $\mathrm{K}$ bajo conjunción. Pero la aparición de la modalidad cambia el juego. Ya no se trata de una teoría empírica, sino de una teoría metafísica y especulativa sobre cómo el conocimiento podría ser. Y dado que considero, fiel a la tradición, que las posibilidades metafísicas son a veces difícilmente imaginables o completamente extrañas, creo que las desviaciones del conocimiento pueden caer dentro de estas extrañezas (en la terminología de Beall).

4. Se cumple el principal objetivo: bloquear el argumento de Fitch. En el siguiente modelo, en $w$ sucede que $p \wedge \neg K p$.

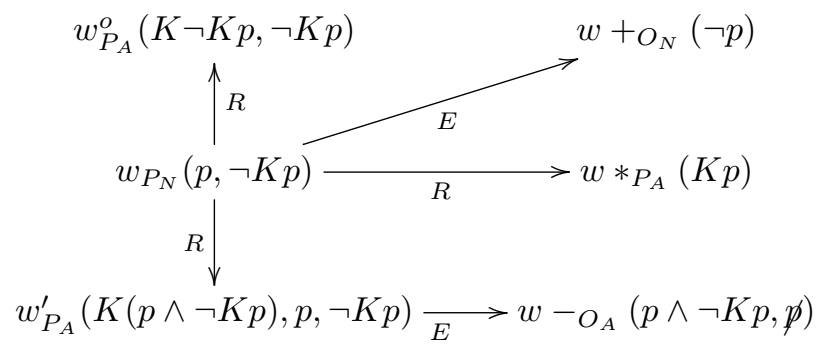

Manuscrito - Rev. Int. Fil., Campinas, v. 37, n. 1, p. 85-116, jan.-jun. 2014. 
Justificación: como había adelantado, aquí se cumple el objetivo de bloquear la argumentación. Eso sucede porque se hace posible conocer $(p \wedge \neg K p)$, gracias a las variaciones epistémicas de los mundos posibles anormales. A diferencia del planteo de Beall (con el cual, obviamente, comparto la idea general), aquí los mundos posibles anormales son simplemente mundos donde los sujetos pueden tener representaciones lógicamente muy pobres del mundo, dando lugar a mundos abiertos de todo tipo de variedad. Por eso ya no son mundos misteriosamente "narcisistas", sino mundos que, como los posibles normales, acceden epistémicamente a mundos abiertos que reflejan los posibles compromisos epistémicos de los sujetos.

5. Sin embargo, si la cognoscibilidad se plantea a la manera tradicional ("todo lo verdadero es cognoscible en un mundo posible normal"), la paradoja sigue valiendo.

Paradoja de Fitch con cognoscibilidad "normal". Supongamos que todo lo verdadero en un mundo posible normal es cognoscible en un mundo posible normal. Sea w un mundo con una verdad desconocida A, es decir, tal que $v_{w}(A \wedge \neg K A)=1$. Por la suposición inicial, hay un mundo posible normal w* tal que wRw* y $v_{w *}(K(A \wedge \neg K A))=1$. Dado que $\mathrm{E}$ es reflexiva, $v_{w *}(A \wedge \neg K A)=1$, por lo cual $v_{w *}(A)=$ $v_{w *}(\neg K A)=1$. Por ende, $v_{w *}(K A)=0$. Pero entonces hay un mundo abierto normal (o posible) $\mathrm{w}+$ tal que $\mathrm{w}^{*} \mathrm{Ew}+\mathrm{y} v_{w+}(A)=0$. Sin embargo, dado que $\mathrm{w}^{*} \mathrm{Ew}+$, tenemos que $v_{w+}(A \wedge \neg K A)=1$, por lo cual $v_{w+}(A)=1$, dado que en los mundos abiertos normales (o en los mundos posibles) valen las cláusulas de la conjunción. Contradicción. 
Justificación: este resultado puede ser insatisfactorio para los anti-realistas. Si la idea es que todo lo verdadero sea cognoscible de la manera en que funciona actualmente el conocimiento, entonces habrá un colapso modal y no podrán existir verdades desconocidas. Sin embargo, creo que refleja un hecho obvio: que el anti-realismo debe ceder en algo frente a la paradoja de Fitch. En la próxima sección explicaré por qué este relajamiento de la cognoscibilidad no es una renuncia ad hoc sino algo que los anti-realistas bien podrían aceptar como aquello que la paradoja "enseña".

\section{Algunos problemas adicionales}

En esta sección, responderé a algunos de los problemas que suscita mi posición $^{11}$.

En primer lugar, admito que, en tanto modelo del conocimiento, lo que presento aquí es al menos incompleto. Por ejemplo, queda mucho por decir acerca de cómo caracterizar con mayor precisión los mundos abiertos normales (es decir, las representaciones de los individuos normales). Hay cierto grado de arbitrariedad en mi planteo: por ejemplo, podemos razonar por introducción de la disyunción pero no siempre podemos conocer las instancias del principio del tercero excluido. Si bien he dado algunos motivos para elegir esa representación y no otra, no me he dedicado en particular a motivar esta particular concepción del conocimiento en mundos posibles normales. La razón principal es que dar una caracterización más realista de la falla de la omnisciencia lógica en nuestro mundo involucra investigaciones que están muy por fuera del alcance de este artículo. De todas maneras, el punto relativo a la paradoja de Fitch, en el que me concentré en este artículo,

\footnotetext{
${ }^{11}$ Agradezco a dos árbitros anónimos por haber selalañado estos puntos problemáticos.
}

Manuscrito - Rev. Int. Fil., Campinas, v. 37, n. 1, p. 85-116, jan.-jun. 2014. 
es independiente de la caracterización particular del conocimiento en mundos posibles normales. Sólo depende de la posibilidad metafísica de agentes lógicamente incompetentes, lo cual he motivado a partir de diversos argumentos filosóficos.

En segundo lugar, como antes señalé, la noción de clausura epistémica que este artículo discute es la siguiente:

Clausura bajo deducción Si S sabe P1,..Pn, y P1,..Pn implican Q, entonces $\mathrm{S}$ sabe $\mathrm{Q}^{12}$

Sin embargo, esta noción no es precisamente la que discuten los epistemólogos de hoy en día. Para muchos epistemólogos, la noción de clausura epistémica que merece ser discutida es la siguiente ${ }^{13}$ :

Clausura bajo deducción realizada Si S sabe P1,..,Pn, y deduce de manera competente $\mathrm{Q}$ a partir de $\mathrm{P} 1, \ldots, \mathrm{Pn}$, llegando a creer que $\mathrm{Q}$ pero reteniendo su conocimiento de que $\mathrm{P} 1, \ldots, \mathrm{Pn}$, entonces S sabe que $Q$.

Ninguno de mis argumentos en el artículo se opone a este tipo de clausura epistémica. De hecho, más allá de los argumentos que nacieron con Dretske (1970), que no están relacionados con la temática de mi artículo, no veo en qué manera algún argumento relativo a la capacidad cognitiva o inferencial de los agentes puede hacer cuestionar el principio de clausura bajo deducción realizada.

Hay un principio distinto de la clausura bajo implicación realizada, y que también ha recibido atención en la bibliografía sobre el tema (por ejemplo, Dretske (2005) utiliza este concepto de clausura):

\footnotetext{
${ }^{12}$ A ciencia cierta, esta es la versión generalizada de la clausura bajo deducción. Parte del debate se centra simplemente en la clausura deductiva bajo razonamientos de una sola premisa.

${ }^{13}$ Hay muchas versiones de este principio, y su correcta formulación es asunto de debate. Aquí tomo la formulación de Hawthorne (2005).
}

Manuscrito - Rev. Int. Fil., Campinas, v. 37, n. 1, p. 85-116, jan.-jun. 2014. 
Clausura bajo implicación conocida Si S sabe $P 1 \ldots P n$ y $\mathrm{S}$ sabe que $\mathrm{P} 1 \ldots . . \mathrm{Pn}$ implican $\mathrm{Q}$, entonces $\mathrm{S}$ sabe $\mathrm{Q}$.

Este principio parece mucho más aceptable que la clausura bajo deducción. Por ejemplo, es razonable decir que si uno sabe que la aritmética de Peano implica A, entonces uno no puede no saber A (presuponiendo que uno conoce los axiomas de la aritmética de Peano). Por otro lado, la clausura bajo implicación conocida es expresable en un vocabulario con conectivos y el operador de conocimiento ${ }^{14}$, a diferencia de la clausura bajo deducción realizada (que apela adicionalmente a la noción de creencia).

Merece llamar la atención que si entendemos $A \rightarrow B$ como $\neg A \vee$ $B$ (como es usual en lógica clásica y en muchas lógicas no-clásicas), entonces un sujeto normal siempre cumple con clausura epistémica bajo implicación conocida (aquí ejemplificaremos con una implicación de una sola premisa, aunque es extendible a casos de más de una premisa). Es decir, en todo mundo posible normal, si $K(A \rightarrow B)$ y $K A$, entonces $K B$.

Prueba: sea w un mundo posible normal donde $K A$ y $K(\neg A \vee B)$ son verdaderas pero $K B$ no lo es. Entonces, debe haber un mundo abierto normal accesible epistémicamente desde $\mathrm{w}$, llamado $\mathrm{w}^{*}$, donde $\neg A \vee B$ es verdadera, $A$ también, pero $B$ no lo es. Por las cláusulas de los conectivos en los mundos abiertos normales, $\neg A$ debe ser verdadero en $\mathrm{w}^{*}$ o $B$ debe serlo. $\mathrm{Si} \neg A$ es verdadero, entonces $A$ no es verdadero en $\mathrm{w}^{*}$. Esto es absurdo. Por silogismo disyuntivo, $B$ debe ser verdadero en $\mathrm{w}^{*}$. Contradicción.

Sin embargo, es fácil ver que la clausura epistémica bajo implicación conocida puede fallar en mundos posibles anormales. Dejo la

\footnotetext{
${ }^{14}$ Donde el concepto de "implicación", ciertamente oscuro en el debate sobre clausura epistémica, es entendido a partir del condicional.
} 
construcción de un contraejemplo al lector.

Lo que podía verse como un problema ahora puede entonces verse como una ventaja. Si bien la clausura bajo deducción falla tanto en mundos posibles normales como anormales, la clausura bajo implicación conocida solamente puede fallar en mundos posibles anormales.

En tercer lugar, hay otras nociones epistémicas en donde la falla de la clausura resulta difícil de aceptar, y esto podría usarse para reformular la paradoja de Fitch. Para describir esta nueva noción epistémica, debo primero decir algo sobre la diferencia entre justificación doxástica y justificación proposicional en teorías de la justificación. Si bien no hay consenso acerca de estos conceptos, en términos generales se sostiene que un agente tiene justificación proposicional de que $\mathrm{p}$ cuando su evidencia total apoya p (véase por ejemplo Neta (2007, p. 197)). En cambio, una creencia de que $p$ está doxásticamente justificada siempre y cuando esté basada en esa evidencia. Por ejemplo, luego de escuchar un juicio donde se dieron pruebas fehacientes de la culpabilidad del acusado, los jueces están proposicionalmente justificados a creer que el acusado es culpable. Esto puede suceder incluso si no creen que el acusado es culpable. Si tienen esa creencia basados en las pruebas disponibles, entonces su creencia está doxásticamente justificada.

Un concepto similar al de justificación proposicional podría sostenerse para teorías del conocimiento. A este concepto podemos llamarlo "estar en posición de saber p". Por ahora, asumamos la siguiente definición de estar en posición de saber p:

(1) S está en posición de saber p si y sólo si S tiene evidencia con la cual podría inferirse $\mathrm{p}^{15}$.

El problema que surge es el siguiente: si entendemos la expresión Kp

\footnotetext{
${ }^{15}$ He intentado dar una definición lo más sencilla posible. Como muestro más adelante, esta definición de estar en posición de saber podría precisarse de distintas maneras. Vale aclarar que la noción de "inferencia" pretende ser general, y no se reduce a la inferencia deductiva.
}

Manuscrito - Rev. Int. Fil., Campinas, v. 37, n. 1, p. 85-116, jan.-jun. 2014. 
en la prueba de la paradoja como "S está en posición de saber p", entonces es muy difícil de entender cómo $\mathrm{K}$ podría no estar cerrado bajo deducción. Pues la evidencia para una proposición constituye también evidencia para sus consecuencias lógicas. Incluso funcionaría en otros mundos posibles: en el caso que nos interesa, es claro que la evidencia para $A \wedge B$ necesariamente es evidencia para $A$.

Asimismo, la correspondiente reformulación del principio de cognoscibilidad parece aceptable desde el punto de vista anti-realista:

Cognoscibilidad proposicional Si p es verdad, entonces un sujeto podría estar en posición de saber que p.

Sin embargo, aquí también la omnisciencia absoluta sería inaceptable: hay muchas verdades cognoscibles sobre las cuales nadie está en posición de saber que son ciertas (por ejemplo, verdades sobre el futuro sobre las cuales no hay evidencia actualmente ${ }^{16}$ ). En resumen, tenemos un nuevo principio de cognoscibilidad que utiliza un operador $\mathrm{K}$ factivo y cerrado necesariamente bajo conjunción, pero donde el operador $\mathrm{K}$ no podría aplicarse a todas las verdades. Por ende, podemos reformular el argumento de Fitch, donde K ya no significa saber sino estar en posición de saber.

¿Qué tiene mi posición para decir sobre esta reformulación de la paradoja de Fitch? En primer lugar, para dar una respuesta a este problema, hace falta hilar fino en el concepto de "estar en posición de saber". El principio que llamé (1) es demasiado fuerte, pues (como señalé) nos compromete con la clausura bajo deducción (excepto quizás en casos como la paradoja de la lotería, que elijo no mencionar por razones de espacio). En cierto sentido, la noción de estar en posición de saber, tal como es caracterizada en (1), involucra cierta referencia implícita a un agente ideal. Es decir, podríamos reformular (1) del siguiente modo:

\footnotetext{
${ }^{16}$ Agradezco a un árbitro anónimo por sugerir este ejemplo.
} 
(1*) S está en posición de saber p si y sólo si tiene evidencia a partir de la cual un agente ideal podría inferir p.

Por eso no se corresponde con nuestras intuiciones respecto al concepto de "estar en posición de saber". Por ejemplo, intuitivamente, saber los axiomas de Peano no lo hace a uno estar en posición de saber todos los teoremas de la aritmética.

Por razones como esas, autores como Sosa (1991, p. 26-29) y Turri (2010) sostuvieron que un correcto análisis de la noción de "estar en posición de saber" debería apelar a cierta normalidad. Puede que uno sepa los axiomas de la artimética pero no esté en posición de saber todos sus teoremas; pero si uno sabe que hace frío y que "si hace frío, es invierno", entonces uno está en posición de saber que es invierno. Una manera de entender esta demarcación para el concepto de "estar en posición de saber" es la siguiente:

(2) S está en posición de saber p siempre y cuando tiene evidencia a partir de la cual un agente normal puede inferir p.

Con la consiguiente reformulación de la cognoscibilidad:

Cognoscibilidad proposicional normal Si p es verdad, entonces un sujeto puede tener evidencia a partir de la cual un agente normal podría inferir $\mathrm{p}$.

Por supuesto, esta reformulación no permite resolver la nueva paradoja de Fitch, pues sigue sin quedar claro cómo alguien puede estar en posición de saber $A \wedge B$ pero no $A$. Claramente, un ser humano normal es típicamente capaz de inferir de acuerdo a la eliminación de la conjunción, por lo cual la evidencia que usa para establecer $A \wedge B$ puede también usarla para establecer A.

Por lo tanto debemos buscar otra reformulación del concepto de "estar en posición de saber". Vale señalar que no siempre relativizar 
la noción de estar en posición de saber a agentes normales arroja resultados intuitivos. Turri (2010, p. 324) ejemplifica este hecho con un agente con una capacidad lógica sobrehumana. En ese caso, si le mostramos un conjunto de axiomas de una teoría, intuitivamente el agente está en posición de saber muchos más teoremas de la teoría que los que una persona normal está en posición de saber. Por ende, (2) no puede explicar el concepto de estar en posición de saber en este caso, y en casos similares.

Una opción razonable para abarcar todos los casos es relativizar de manera totalmente específica:

(3) S está en posición de saber p siempre y cuando tiene evidencia a partir de la cual S mismo podría inferir que p.

El mismo Hawthorne (2005, p. 31) da una versión similar para el caso de la distributividad del conocimiento bajo conjunción, donde uno está en posición de saber $\mathrm{P}$ siempre y cuando uno sea capaz de deducirlo:

(Distribución) Si uno sabe la conjunción entre P y Q, entonces en la medida en que uno sea capaz de deducir $\mathrm{P}$, uno está en posición de saber $\mathrm{P}$ (y en la medida en que uno sea capaz de deducir Q, entonces uno está en posición de saber Q)

A partir de (3), el principio de cognoscibilidad proposicional nos diría algo como esto:

Cognoscibilidad proposicional específica Si p es verdad, entonces un agente podría tener evidencia que él mismo podría usar para inferir p.

Mi posición puede comprometerse con (3) y con este último sentido de cognoscibilidad proposicional. Vale la pena señalar que respecto a la falla en la eliminación de la conjunción, mi posición apela simplemente a agentes metafísicamente posibles. $\mathrm{Y}$ un agente meramente posible 
podría ser lógicamente muy incompetente. Si ese es el caso, entonces es concebible que esté en posición de saber $A \wedge B$ pero no esté en posición de saber $A$. Simplemente el agente es capaz de utilizar la evidencia de la que dispone para inferir $A \wedge B$, pero (en parte debido a su incapacidad de deducir $A$ a partir de $A \wedge B$ ) es incapaz de utilizarla para inferir $A$.

\section{Conclusión: Una lección rara de la para- doja de Fitch}

El desafío planteado por la paradoja de Fitch es una genuina paradoja para el anti-realista, y lo obliga a hacer algo para salvarse. Como antes señalé, el anti-realismo, para no caer en finitismo estricto, debe postular siempre la posibilidad de conocedores finitos con capacidades naturales muchísimo mayores a las de un ser humano. Esto es, debe idealizar. Sin embargo, la paradoja de Fitch muestra que incluso estos conocedores idealizados no pueden conocer algunas verdades.

En mi posición se da una idealización en sentido inverso: algunos conocedores tienen capacidades muchísimo menores a las de un ser humano normal. Esto permite que, por ejemplo, conozcan una conjunción pero no los conyuntos. Esto puede suceder, por ejemplo, porque no se dan cuenta que la conjunción los implica. Este fenómeno es una rareza, y de hecho contradice las verdades de la lógica epistémica. Sin embargo, en mi teoría es visto como otra entre tantas posibilidades metafísicas que contradicen leyes empíricas (dado que las leyes epistémicas no son más que leyes empíricas, según mi perspectiva).

El factor que modifica los asuntos es la posibilidad. Especialmente porque en mi teoría la lógica es clásica en todo mundo posible, por lo cual valen todas las verdades lógicas, y las leyes epistémicas son tautologías, porque valen en los mundos posibles normales. Sólo en 
mundos posibles anormales pueden fallar las leyes epistémicas.

¿Puede el anti-realista aceptar una posición como esta? Creo que sí. Pues la idealización inversa no tiene ningún rasgo extraño, si se acepta la idealización tradicional. Puede objetarse que la idealización tradicional no rompe con ninguna regla de racionalidad (como las leyes epistémicas), mientras que la inversa sí lo hace. Esa parece ser la diferencia fundamental entre los dos tipos de idealización. Sin embargo, una vez que rompemos con la ilusión de la clausura epistémica, la diferencia entre el humano normal y el inversamente idealizado es meramente de grado. Quizás, ciertamente, el segundo pierda algunos componentes de racionalidad (parece que la aplicación general de leyes $\mathrm{K}$ es bastante fundamental); sin embargo, su diferencia puede ser, en sentido estricto, intrascendente. Por ejemplo, puede que cumpla con todas las leyes $\mathrm{K}$, pero no aplique la distributividad sólo en el caso de la oración de Fitch. Eso no puede transformarlo directamente en irracional, sino como mucho, en otro ser racional con algunas fallas epistémicas. Su mundo epistémico puede ser ligeramente más abierto que el normal, lo cual le permita conocer algunas cosas que los conocedores más ágiles lógicamente no pueden saber. Quizás se encuentre aquí el hecho paradójico: en este caso particular, menos "racionalidad" posibilita más conocimiento.

Todo lo verdadero, entonces, es cognoscible (puede decir el antirealista). No necesariamente cognoscible por un conocedor ideal, o por un conjunto de conocedores ideales. Sino por distintos sujetos, algunos epistémicamente ideales, otros epistémicamente normales, y otros ideales en sentido inverso, es decir, con un poco menos de capacidad lógica que los normales ${ }^{17}$.

\footnotetext{
${ }^{17}$ El punto es similar al de Restall (2009), quien defiende que todo lo verdadero es cognoscible en un sentido ligeramente desviado: que una conjunción sea declarada cognoscible cuando ambos conyuntos pueden ser conocidos. Sin embargo, el problema de su propuesta es que justamente toca el principio de cognoscibilidad, y algunas verdades dejan de ser, en sentido estricto,
} 


\section{Referencias}

BEALL, JC. Knowability and possible epistemic oddities, en Salerno (ed.), (2009).

DRETSKE, F. "Epistemic operators", Journal of Philosophy 67: pp. 1007-1023, 1970.

—_. "Is knowledge closed under known entailment?", en M. Steup y E. Sosa (eds.), Contemporary Debates in Epistemology, Blackwell, (2005).

DUMMETT, M. Fitch's paradox of knowability, en Salerno (ed.), (2009).

EDGINGTON, D. The Paradox of Knowability, Mind 94: pp. 557-68, 1985.

FIRTH, R. "Are Epistemic Concepts Reducible to Ethical Concepts?" en Goldman y Kim (eds.) Values and Morals, Dordrecht: D. Reidel Publishing Co., (1978).

GOLDMAN, A. I. \& KIM, I. Values and Morals, Dordrecht: D. Reidel Publishing Co., 1978.

HAWTHORNE, J. "The case for closure", en M. Steup y E. Sosa (eds.), Contemporary Debates in Epistemology, Blackwell, (2005).

JENKINS, C. The Mystery of the disappearing diamond, en Salerno (ed.), (2009).

KVANVIG, J. Restriction strategies for Knowability: some lessons in false hope, en Salerno (ed.), (2009).

—_. "Closure Principles", Philosophy Compass 1(3): pp. 256-267, 2006.

PRIEST, G. Towards non-being, OUP, 2006.

—. Beyond the limits of Knowledge, en Salerno (ed.), (2009).

- An introduction to non-classical logic, Oxford University Press, 2006.

cognoscibles.

Manuscrito - Rev. Int. Fil., Campinas, v. 37, n. 1, p. 85-116, jan.-jun. 2014. 
RESTALL, G. Not every truth can be known (at least, not all at once), en Salerno (ed.), (2009).

SALERNO, J Introduction, en Salerno (ed.), (2009).

. (ed.) New essays on the Knowability Paradox, Oxford University Press, 2009.

. Knowability and possible epistemic oddities, Oxford University Press, 2009.

- Fitchs paradox of Knowability, Oxford University Press, 2009.

- The Mystery of the disappearing diamond, Oxford University Press, 2009.

- Restriction strategies for Knowability, Oxford University Press, 2009.

- Beyond the limits of Knowledge, Oxford University Press, 2009.

—. Not every truth can be known, Oxford University Press, 2009.

—. Tennants Troubles, Oxford University Press, 2009.

SOSA, E. Knowledge in perspective, Cambridge University Press, 1991.

STALNAKER, R. Inquiry, MIT Press, 1984.

STEUP M. \& SOSA E. Contemporary Debates in Epistemology, Blackwell, 2005.

TENNANT, N The taming of the true, Oxford University Press, 1997.

TURRI, J. "On the relationship between doxastic and propositional justification", Philosophy and Phenomenological Research, 80(2): pp. 312-326, 2010.

WILliAMSON, T. Tennant's Troubles, en Salerno (ed.), (2009).

- Knowledge and its limits, Oxford University Press, 2000.

WRIGHT, C. "Strict Finitism", Synthese 51 (2): pp. 203 - 282, 1982. 